\title{
Determinação do nível de gravidade do trauma*
}

\author{
Determination of trauma severity level by means of the injury severity score
}

\author{
Terezinha Dalossi Gennari, Maria Sumie Koizumi \\ Unidade de Terapia Intensiva da Casa da Saúde Dr. Domingos Anastácio - \\ Jundial, SP - Brasil (T.D.G.), \\ Departamento de Enfermagem Médico-Cirúrgica da Escola de Enfermagem da Universidade de Sāo \\ Paulo - São Paulo, SP - Brasil (M.S.K.)
}

\begin{abstract}
Trata-se de estudo prospectivo que teve por objetivo caracterizar a gravidade do trauma de pacientes hospitalizados, através do "Injury Severity Score" (ISS). Forarm analisados 100 pacientes de trauma internados em uma instituiçăo referência para trauma locallzada em São Paulo, Brasil. Do total de paclentes, 68 sofreram trauma fechado 032 trauma penetrante. Dentre os pacientes de trauma fechado, $53,0 \%$ sofreram trauma leve (ISS $1-15$ ), $29,4 \%$ trauma moderado (ISS 16-24) e 17,6\% trauma grave (ISS $\geq 25$ ) enquanto que $34,4 \%$ dos pacientes de trauma penetrante sofreram trauma leve, $18,7 \%$ trauma moderado e $46,9 \%$ trauma grave. A média e desvio-padrăo dos ISSs dos pacientes de trauma fechado e penetrante foi, respectivamente, de $14,9 \pm 8,1$ e $20,8 \pm 11,0$, correspondendo a um percentual de mortos de $11,8 \%$ e $12,5 \%$.
\end{abstract}

Escala de gravidade do ferimento. Ferimentos e lesōes, prevençāo \& controle..

\section{Intrơuçäo}

Nos últimos vinte anos, vários índices foram desenvolvidos e vêm sendo refinados para determinar a gravidade do trauma, tendo como base critérios

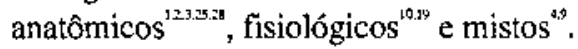

Por meio de uma linguagem uniforme, os índices de gravidade permitem, dentre outros aspectos, a comparação da morbidade e mortalidade entre grupos de pacientes cuja gravidade do trauma seja similar. Favorecem, desta forma, a avaliação e a comparação da assistência prestada por diversos serviços de atendimento ao traumatizado, o acompanhamento da evolução da assistência ao longo do tempo, bem como a efetividade das medidas instituídas ${ }^{3.1 .11 .13 .19 .16}$. Os índices podem também auxiliar no processo de triagem dos pacientes, no planejamento de serviços, na distribuiçāo de recursos humanos e materiais e na

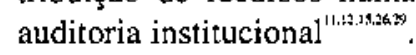

Dentre os índices desenvolvidos para determinar a gravidade do trauma de pacientes hospitalizados, o "Injury Severity Score" (ISS), derivado da "Abbreviated Injury Scale" (AIS), de base anatômica, tem sido reconhecido como o mais promissor, por ser dotado de sensível poder prognóstico com vistas à sobrevivência e mortalidade s.1.1.2.130. $^{\text {s. }}$.

A AIS é um sistema que classifica lesões de acordo com o tipo e gravidade em una escala ordinal de 6 pontos. Foi desenvolvida nos Estados Unidos da América e publicada pela primeira vez em 1971. Posteriormente, nos anos de $1974,1976,1980,1985$ e 1990, foi revisada com vistas ao seu refinamento ${ }^{1,21427}$. Em 1976 foi apresentada, pela primeira vez, sob a forma de um manual com cerca de 500 descrições de lesōes'.". Em todas as revisōes, foram classificadas e acrescentadas descriçōes. A AIS-80 reunia mais de $500^{n}$, a AIS-85 mais de $1.200^{132}$ e a 
versão mais atual, congrega acima de 2.000 descriçōes de lesōes'.

Cada lesão contida no Manual AIS pode receber valores de 1 a 6 . Escore 1 é atribuído à injúria de gravidade leve, 2 à moderada, 3 à grave que não ameaça a vida, 4 à grave que ameaça a vida, porém com sobrevivência provável, 5 à crítica, com sobrevivência incerta, e 6 para a lesão máxima que é quase sempre fatal.

O ISS foi desenvolvido em 1974, a partir da AIS, por Baker e col., e é definido como um escore obtido matematicamente a partir da soma dos quadrados dos escores AIS mais altos de três regiões diferentes do corpo, mais gravemente traumatizadas'. Pelo ISS obtém-se o escore de gravidade do trauma, que é global.

As seis regiões do corpo usadas para computar o ISS são: cabeça ou pescoço; face; tórax; abdome ou conteúdos pélvicos; membros superiores, inferiores ou cintura pélvica e superfície externa.

A pontuação do ISS varia de 1 a 75 . Os escores mais altos indicam maior gravidade do trauma e mais elevada $\varepsilon$ a probabilidade de morte. O ISS máximo de um paciente que apresenta lesōes em apenas uma região do corpo é $25\left(5^{3}\right)$. O paciente terá ISS de 75 quando apresentar lesões de escore AIS 5 e em três regiões do corpo $\left(5^{2}+5^{2}+5^{2}\right)$. Por definiçāo, o ISS é igual a 75 para qualquer pessoa que tenha uma lesão de escore AIS 6. Um escore ISS > 16 tem sido considerado como aquele de ponto crítico ${ }^{6.11 .6 .29}$.

Tendo em vista a importância do conhecimento dos escores de gravidade do trauma (ISSs) dos pacientes, ainda durante a internação hospitalar, bem como a complexidade crescente do Manual AIS, dificultando seu uso rotineiro em unidades que assistem essas vítimas, diversos autores idealizaram mapas que resumem ou simplificam o Manual AIS, objetivando pontuações mais rápidas das lesōes e agilização no cálculo dos ISSs ${ }^{13: 2027}$.

Dentre esses mapas, o "Condensed Abbreviated Injury Scale" (CAIS-85), elaborado por Civil \& Schwab:" nos Estados Unidos da América, a partir do Manual AIS-85, é o mais desenvolvido.

O CAIS-85 são dois mapas que condensam as lesőes dos pacientes de trauma fechado (CAIS$85 \mathrm{~F}$ ) e as dos pacientes de trauma penetrante (CAIS-85P). Estas lesões são apresentadas separadamente nas seis regiões do corpo utilizadas para computar o ISS, e de acordo com os escores de gravidade AIS.

A escassez de estudos no país sobre a quantificação da gravidade do trauma por meio de índices de gravidade, levou a realização do presente estu- do que teve por objetivo caracterizar a gravidade do trauma de pacientes intemados, atraves do "Injury Severity Score", fornecendo subsídios para as áreas de prevençâo, assistência, ensino e pesquisa do trauma.

\section{Material e Método}

A populaçāo de estudo foi constituída por 100 vítimas de trauma internadas no período de março a julho de 1992, em hospital municipal de referência para trauma, localizado em Sāo Paulo, Brasil.

Foram critérios para inclusão desses pacientes:

(a) terem sido admitidos via pronto-socono por lesão traumática decorrente de acidente de trânsito de veículo a motor, tentativa de homicídio, queda acidental e tentativa de suicídio;

(b) serem provenientes diretamente do Iocal do evento ou de outros hospitais desde que não tivessem sofrido cirurgias e o trauma tivesse ocorrido no máximo um dia atrás;

(c) terem permanecido no hospital por um período de tempo $\geq 24$ horas; $\mathrm{e}$

(d) terem idade $\geq 12$ anos.

Os pacientes que foram transferidos para outros hospitais foram excluídos do estudo.

A identificação da lesão teve como fonte principal o prontuário do paciente. Além disso, foi realizada por uma das autoras uma inspeção física em todos os pacientes para identificar as lesões da superfície externa, já que nem sempre eram anotadas pelos médicos no prontuário. Também foi realizada uma entrevista com todos os pacientes para determinar a ocorrência ou não de amnésia pós acidente, pois esta informação é verificada e apenas eventualmente constava no prontuário do paciente.

Identificadas todas as lesoes do paciente, assinalou-se sua gravidade por meio de consulta aos mapas CAIS-85F (Anexo 1) e CAIS-85P (Anexo 2), utilizados na presente pesquisa, de acordo com as recomendaçס̃es daqueles que os construíram. Logo, as lesōes dos pacientes de traumna fechado foram verificadas através de consulta ao mapa CAIS-85F e as lesões dos pacientes de trauma penetrante receberam escores por meio de consulta ao mapa CAIS-85P. O Manual AIS-85 foi consultado para atribuir escores apenas para as lesões não listadas nos mapas CAIS-85. 
Após a marcação das lesões de todos os pacientes, procedeu-se ao cálculo da gravidade do trauma. Neste ponto, as lesões identificadas foram classificadas segundo as seis regiôes do corpo usadas para o cálculo do ISS. A seguir, para cada paciente do estudo foi assinalado o escore AIS mais alto de cada uma das seis regiôes corpóreas. Assim sendo, a gravidade do trauma foi obtida pela somatória dos quadrados do escore AIS mais alto de três regiōes diferentes do corpo mais gravemente traumatizadas e expressadas como ISS de marcação 1 a 75.

Com relação ao ISS, os pacientes foram subdivididos em 3 grupos, de 1 a 15,16 a 24 e $\geq 25$. Os pacientes inseridos no intervalo de ISS de 1 a 15 foram classificados como pacientes de trauma leve, de 16 a 24 como trauma moderado, e $\geq 25$ como trauma grave. A marcaçāo $\geq 16$ foi adotada como ponto crítico ${ }^{4,21,26,29}$.

\section{Resultados e Discussão}

\section{Caracterização dos Pacientes quanto à Causa Externa, Tipo de Trauma, Idade e Sexo}

Um total de 40 pacientes $(40,0 \%)$ foram vítimas de acidente de trânsito de vé́culo a motor, 39 $(39,0 \%)$ de -tentativa de homicídio, $19(19,0 \%)$ de queda acidental e $2(2,0 \%)$ de tentativa de suicídio.

O mecanismo do acidente ou violência que causou o trauma foi do tipo fechado em 68 pacientes $(68,0 \%)$ e penetrante em $32(32,0 \%)$.

A idade dos pacientes de trauma fechado variou de 13 a 70 anos, sendo que a média foi de 35,4 anos. Um total de 57 pacientes $(83,9 \%)$ tinham idade de 13 a 50 anos. Quanto ao sexo, houve predomínio do masculino $(80,9 \%)$.

A idade dos pacientes de trauma penetrante variou de 16 a 55 anos e a média foi de 27,2 anos, bem inferior a dos pacientes de trauma fechado. $O$ trauma penetrante atingiu basicamente pessoas de 13 a 40 anos $(93,8 \%)$ e do sexo masculino $(93,8 \%)$.

O predomínio de adultos jovens do sexo masculino tem sido registrado em muitos estudos que envolvem acidentes e violências de natureza traumática ${ }^{8,17,22,33}$ e se confirmou verdadeiro também na presente investigação.

Quanto à causa externa, como já foi demonstrado por outros autores ${ }^{8,14,17,18,21}$, o acidente de trânsito de veículo a motor ficou situado em primeiro lugar. Além disso, ressalte-se o fato de que as tentativas de homicídio ficaram muito próximas aos acidentes de trânsito. Isso mostra o homicídio, não só como uma importante causa de mortalidade mas, provavelmente, também, de morbidade, no Município de São Paulo.

\section{Caracterização dos Pacientes segundo a Gravidade das Lesões (AIS) e Gravidade do Trauma (ISS)}

Um total de 323 lesões anatômicas foram diagnosticadas nos 100 pacientes do estudo, desde a entrada no hospital até a alta ou óbito, em média 3,2 lesôes por paciente.

Nos pacientes de trauma fechado ocorreram 223 lesőes anatômicas, perfazendo uma média de 3,3 , por paciente. Além dessas, foram constatadas 66 descrições nāo-anatômicas, referentes a alteraçōes do nível de consciência e período de inconsciência nas primeiras 24 horas após o trauma.

As lesões de gravidade leve (AIS ${ }_{1}$ ), moderada $\left(\mathrm{AIS}_{2}\right)$ e grave, que não ameaçam a vida ( $\mathrm{AIS}_{3}$ ), foram as mais comuns entre os pacientes de trauma fechado, totalizando $86,5 \%$ das lesões. Embora as lesões de escores AIS 4 e 5 nāo tenham sido tāo frequientes $(13,5 \%)$, é importante realçá-las pelo fato de que lesōes de escores AIS 4 ameaçam a vida e na presença de lesões AIS; a sobrevivência é incerta.

O total de 100 lesōes ocorreram nos 32 pacientes de trauma penetrante, em média 3,1 por paciente. A gravidade das lesões dos pacientes de trauma penetrante foi diferente. Ela foi principalmente moderada ( $\mathrm{AIS}_{2}$ ) e grave, sem e com ameaça a vida (AIS 3 e 4), englobando $76,0 \%$ do total de lesōes. O número de lesôes de escores AIS 4 e 5 , proporcionalmente ao número de pacientes, foi maior nos pacientes de trauma penetrante.

A caracterização dos pacientes, segundo a gravidade do trauma, apresentada a seguir, foi realizada com base nos ISSs calculados à luz dos diagnósticos finais, por ocasião da alta ou óbito.

Nos pacientes de trauma fechado os ISSs variaram de 1 a 42 , sendo que a média e desviopadrão foi $14,9 \pm 8,1$. Como mostra a Tabela 1 , dos 68 pacientes de trauma fechado, $36(53,0 \%)$ sofreram trauma leve (ISS de 1 a 15), $20(29,4 \%)$ trauma moderado (ISS de 16 a 24) e $12(17,6 \%)$ trauma grave (ISS 225 ). Do total de pacientes de trauma fechado, $32(47,0 \%)$ sofreram trauma importante, definido por um ISS igual ou maior que 16.

Em relação às condiçōes de saída do hospital, 60 pacientes $(88,2 \%)$ receberam alta e $8(11,8 \%)$ morreram. Os ISSs referentes aos pacientes que morreram foram $9,10,16,17,17,25,26$ e 30 . A faixa etária de 7 desses pacientes era de 21 a 50 anos e apenas uma paciente tinha 68 anos. 
Tabela 1- Distribuição dos pacientes de trauma fechado, segundo intervalo de ISS e condiçă̌o de saida do hospltal, HE-31, 1992.

\begin{tabular}{|c|c|c|c|c|c|c|}
\hline \multirow{2}{*}{$\begin{array}{l}\text { Condiçāo } \\
\text { ISS }\end{array}$} & \multicolumn{2}{|c|}{ Alta } & \multicolumn{2}{|c|}{ Sbito } & \multirow{2}{*}{$\begin{array}{l}\text { Total } \\
\mathrm{N}\end{array}$} & \multirow{2}{*}{$\%$} \\
\hline & $N$ & $\%$ & $N$ & $\%$ & & \\
\hline $\begin{array}{l}01-15 \\
16 \mid-24 \\
25 \text { ou + }\end{array}$ & $\begin{array}{l}34 \\
17 \\
09\end{array}$ & $\begin{array}{l}(50,0) \\
(25,0) \\
(13,2)\end{array}$ & $\begin{array}{l}02 \\
03 \\
03\end{array}$ & $\begin{array}{l}(3,0) \\
(4,4) \\
(4,4)\end{array}$ & $\begin{array}{l}36 \\
20 \\
12\end{array}$ & $\begin{array}{l}(53,0) \\
(29,4) \\
(17,6)\end{array}$ \\
\hline Total & \multicolumn{2}{|c|}{$60(88,2)$} & \multicolumn{2}{|c|}{$08(11,8)$} & \multicolumn{2}{|c|}{$68(100,0)$} \\
\hline
\end{tabular}

ISS - "Injury Severety Score"

$\bar{X}$ do ISS $=14,9 \pm 8,1$

Especificamente em relaçāo aos pacientes de trauma penetrante, os ISSs variaram de 4 a 41 e a média foi $20,8 \pm 11,0$, superior aos pacientes de trauma fechado.

De acordo com a Tabela 2 , dos 32 pacientes de

Tabela 2- Distribuiçāo dos pacientes de trauma penetrante segundo intevalos de ISS a condição de saida do hospital, HE-31, 1992.

\begin{tabular}{lllll}
\hline Condição & Alta & \multicolumn{2}{l}{ Obito } & \multicolumn{2}{l}{ Total } \\
\hline ISS & $\mathrm{N} \%$ & $\mathrm{~N} \%$ & $\mathrm{~N} \mathrm{\%}$ \\
\hline O1 $|-| 15$ & $11(34,4)$ & $\ldots \ldots \ldots \ldots . .$. & & $11(34,4)$ \\
$16|-| 24$ & $06(18,7)$ & $\ldots \ldots \ldots \ldots$. & $06(18,7)$ \\
25 ou + & $11(34,4)$ & $04(12,5)$ & $15(46,9)$ \\
\hline Total & $28(87,5)$ & $04(12,5)$ & $32(100,0)$ \\
\hline
\end{tabular}

ISS - "Injuri Severety Score"

$\bar{X}$ do $1 S S=20,8 \pm 11,0$

trauma penetrante, $11(34,4 \%)$ foram levemente traumatizados, $6(18,7 \%)$ moderadamente e 15 $(46,9 \%)$ gravemente traumatizados. A gravidade do trauma desses pacientes foi mais alta se comparada a dos pacientes de trauma fechado, uma vez que 21 $(65,6 \%)$ tiveram um ISS igual ou maior que 16.

Um total de 28 pacientes $(87,5 \%)$ sobreviveram e $4(12,5 \%)$ morreram. Os ISSs dos pacientes que faleceram foram $35,36,41$ e 41 , sendo todos da faixa etária de 13 a 40 anos.

A análise do total de pacientes do presente estudo, independente do tipo de trauma, Tabela 3, mostra que $47(47,0 \%)$ sofreram trauma leve, 26 $(26,0 \%)$ trauma moderado e $27(27,0 \%)$ trauma grave. A média do ISS de todos os pacientes foi $16,8 \pm 9,5$, correspondendo a um percentual de mortos de $12,0 \%$.

Além de Baker e col. ${ }^{3}$, outros validaram o ISS em grandes séries de pacientes de idade superior a doze anos, tanto de trauma fechado como penetrante $^{5.30}$. Mais recentemente Copes e col. ${ }^{14}$ validaram o ISS, usando a AIS-85. Os resultados desses estudos, bem como os de outros ${ }^{7.8 .17 .26 .29 .31}$, têm confirmado a correlação do ISS com mortalidade, verificada inicialmente por Baker e col. ${ }^{3}$

Coincidindo com a literatura internacional, os dados da Tabela 3 mostram que, apesar do número

Tabela 3- Distribuiçāo dos pacientes de trauma fechado e penetrante, segundo intervaios de ISS e condiçấo de saida do hospital, HE-31, 1992.

\begin{tabular}{|c|c|c|c|c|c|c|}
\hline \multirow{2}{*}{$\begin{array}{l}\text { Condiçẵo } \\
\text { ISS }\end{array}$} & \multicolumn{2}{|c|}{ Alta } & \multicolumn{2}{|c|}{ Obito } & \multicolumn{2}{|c|}{ Total } \\
\hline & $\mathbf{N}$ & $\%$ & $N$ & $\%$ & $\mathbf{N}$ & $\%$ \\
\hline $\begin{array}{l}01 \text { - } 15 \\
16 \text { - } 24 \\
25 \text { ou }+\end{array}$ & \multicolumn{2}{|c|}{$\begin{array}{l}45(45,0) \\
23(23,0) \\
20(20,0)\end{array}$} & \multicolumn{2}{|c|}{$\begin{array}{l}02(2,0) \\
03(3,0) \\
07(7,0)\end{array}$} & \multicolumn{2}{|c|}{$\begin{array}{l}47(47,0) \\
26(26,0) \\
27(27,0)\end{array}$} \\
\hline Total & \multicolumn{2}{|c|}{$88(88,0)$} & \multicolumn{2}{|c|}{$12(12,0)$} & \multicolumn{2}{|c|}{$100(100,0)$} \\
\hline
\end{tabular}

ISS - "Injury Severety Score"

$\bar{X}$ do ISS $=16,8 \pm 9,5$

de pacientes do presente estudo ser bem menor, à medida que os escores ISS vāo aumentando, há também acréscimos nos percentuais de mortos e o contrário ocorre para aqueles que sobrevivem. De acordo com as expectativas, a grande maioria dos pacientes que morreram, $10(83,3 \%)$ tinham um ISS igual ou maior de 16 e $2(16,7 \%)$ tinham um ISS igual ou menor que 15 . Face ao número reduzido de pacientes que faleceram, somente a título de ilustração, analisou-se os dados constantes nos seus laudos de necropsia.

Dos pacientes que morreram, do intervalo de ISS de 1 a 15 , um era uma mulher de 68 anos que sofreu uma queda e como conseqüência teve apenas o fêmur fraturado (ISS =9). A causa de óbito, segundo laudo da necrópsia realizada no Instituto Médico Legal (IML), foi broncopneumonia no curso do tratamento da fratura de fêmur. Outro paciente que morreu, do mesmo intervalo de ISS, do sexo masculino, de 25 anos, morreu também vítima de queda. Havia descrito em seu prontuário fraturas de crânio e hemorragia subaracnoídea constatadas por tomografia computadorizada e um hematoma periorbital (ISS $=10$ ). Manteve-se com um escore da Escala de Coma de Glasgow de 13, durante as primeiras 24 horas da admissão, tendo posteriormente havido rebaixamento do nível de consciência e evoluçāo rápida para coma Depassé. No relatório de necrópsia do IML constava, além das fraturas de crânio e hematoma de órbita, um hematoma subdural e pneumotórax. Assim, o ISS 
real desse paciente foi 26 , justificando portanto sua evolução para o óbito que ocorreu por Trauma Crânio Encefálico (TCE).

Os três pacientes do intervalo de ISS de 16 a 24 tiveram como causa mortis, pelo relatório de necrópsia do IML, TCE produzido por agente contundente.

Quatro dos sete pacientes do grupo de ISS $\geq 25$ morreram devido a TCE, sendo que em três o óbito foi produzido por agente contundente e em um por agente pérfuro-contundente (projétil de arma de fogo). Os três pacientes restantes tiveram como causa mortis, respectivamente, politraumatismo produzido por agente pérfuro-contundente, hemorragia interna causada por agente pérfuro-contundente e broncopneumonia contraída no decurso de tratamento de lesōes por projétil de arma de fogo.

Assim sendo, analisando as causas das mortes ocorridas na populaçãodo presente estudo, destacou-se o traumatismo crânio-encefálico já que ele foi a causa determinante da morte de 8 dos 12 pacientes. Além do TCE ter sido a principal causa mortis, é importante destacar que dos 100 pacientes, $44(44,0 \%)$ apresentaram lesões anatômicas no crânio ou no conteúdo craniano.

Diversos estudos mostram que a incidência de TCE $\varepsilon$ bastante elevada em populaçōes traumatizadas $^{18,22.23}$, Além disso, o TCE é considerado como um fator dominante na determinação do prognóstico do paciente ${ }^{14.18}$, Gennarelli e col. ${ }^{18}$, estudando 49.143 pacientes traumatizados de 95 centros de trauma, verificaram que $16.524(34 \%)$ sofreram TCE. Destes, $3.061(18,53 \%)$ apresentaram só TCE, $7.058(42,71 \%)$ sofreram, além de TCE. lesões de gravidade leve na superfície extema (AIS 1 e 2 ), e $6.405(38,76 \%)$ sofreram TCE e lesões extracranianas de escores AIS de 3 a 6 . A mortalidade global dos pacientes com TCE foi $18,2 \%$, três vezes mais alta do que a população sem TCE $(6,1 \%)$. Foi verificado também que dos 16.524 pacientes que sofreram TCE, 3.010 morreram. A morte foi atribuída à gravidade da lesão craniana em 2.040 pacientes $(67,8 \%)$, a complicaçōes de lesōes em $25,6 \%$ e a lesões extracranianas em somente $6,6 \%$.

Ainda com relação à gravidade do trauma, vários estudos têm comprovado que o percentual de mortos é baixo em grupos de pacientes de ISS de 1 a 15 , com tendência a aumentar a partir do escore $16^{3.8 .14 .26 .31}$. Além disso, os estudos mostram que trauma de gravidade similar em pessoas mais jovens e mais velhas geram prognóstico pior nestes últimos pacientes ${ }^{337.26,31}$. Long e col. $^{26}$ verificaram que de 2.511 pacientes, 1.839 tiveram ISS $\leq 15$ e $672 \geq 16$. Dos dezenove pacientes $(1,04 \%)$ do grupo de ISS $\leq 15$ e $137(20,3 \%)$ de ISS $\geq 16$ morreram. Todos os pacientes que faleceram de ISS $\leq 15$ eram velhos, de idade entre 64 a 94 anos. Os autores concluíram que pacientes de ISS $\leq 15$ devem ser definidos como de trauma leve e aqueles de ISS $\geq 16$ como de trauma importante.

Champiom e col. ' calcularam as freqüûncias de mortos de 42.944 pacientes de idade $<65$ e de 3.833 de idade $\geq 65$ anos, de 111 centros de trauma dos Estados Unidos da América e Canadá, de acordo com os intervalos de ISS de 0 a 8,9 a 15,16 a 24,25 a 40,41 a 49,50 a 74 e 75 . Constataram que os percentuais de mortos nos pacientes de idade < 65 anos, segundo os intervalos acima citados, foram de $0,3 \%, 2,7 \%, 10,5 \%, 29,3 \%, 50,0 \%$, $65,2 \%$ e $89,9 \%$ e nos pacientes de idade $\geq 65$ anos foram de $2,9 \%, 6,9 \%, 28,9 \%, 51,4 \%, 73,7 \%$, $90,5 \%$ e $94,4 \%$. A média dos escores ISS do grupo de pacientes mais jovens foi 13,4 e a dos idosos foi 13,3 , correspondendo, respectivamente, a um percentual de mortos de $9,8 \%$ e $19,0 \%$.

Smith e col, ${ }^{31}$ compararam as freqüências de mortos de 985 pacientes de idade $<65$ anos com as de 456 pacientes de idade $\geq 65$ anos, de acordo com os intervalos de ISS de 1 a 15,16 a 25 e $>25$. Os percentuais de mortos do grupo de pacientes mais jovens, nesses intervalos de ISS, foram $0,4 \%$, $12,1 \%$ e $28,6 \%$ e para o grupo de idosos foram $2,1 \%, 21,6 \%$ e $62,2 \%$. A mortalidade global no grupo dos mais jovens e dos idosos foi, respectivamente, de $6,0 \%$ e $8,6 \%$. A média do ISS dos idosos foi $10,8 \pm 9,1$.

Copes e col. ${ }^{14}$ calcularam a freqüência de mortos para cada escore ISS de 8.629 pacientes de idade $<50$ anos e 2.544 de idade $\geq 50$ anos com trauma fechado. O mesmo foi feito para 3.424 pacientes ( $<50$ anos) e 279 ( $\geq 50$ anos) com trauma penetrante. Analisando os quadros de distribuição dos pacientes do presente estudo, independente da idade, a taxa de mortos entre pacientes do grupo de ISS de 1 a 15 oscilou em torno de $1,0 \%$, tanto para pacientes de trauma fechado como de trauma penetrante. No grupo de ISS de 16 a 24 a mortalidade foi de $12,5 \%$ para os pacientes de trauma fechado e $9,4 \%$ para os de trauma penetrante. A partir do escore 25 a mortalidade aumentou de forma quase linear.

Hill e col. ${ }^{21}$ constataram que uma média de ISS de 22,6 $\pm 13,4$ relacionou-se com uma percentagem de mortos de $14 \%$ em um grupo de 544 pacientes de uma UTI cirúrgica de Montreal no Canadá.

Schwab e col. ${ }^{29}$, usando o CAIS-80 para o cálculo do ISS, constataram percentagem de mortos de 
$6.3 \%$ em uma população de 523 pacientes de trauma, cuja média dos ISSs foi de 15.16. A frequiência de mortos, de acordo com os intervalos de ISS de 1 a 8,9 a 15,16 a 24,25 a 40 e $\geq 41$, foi, respectivamente, de $0 \%, 0,01 \%, 3,8 \%, 15,1 \%$ e $40,5 \%$.

Frutiger e col. ${ }^{17}$, estudando 233 pacientes de uma UTI cinúrgica cujos ISSs, com base na AIS-80, eram > 18 e média de 29,3 , verificaram percentagem de mortos de $18,4 \%$.

$\mathrm{Na}$ literatura intemacional, há tendência em comparar resultados de pesquisas de populaçōes contemporâneas de trauma fechado com aqueles constatados por Baker e col." ou Bull'. Entretanto, para alguns pesquisadores esses dados são incomparáveis, uma vez que as lesões estudadas pelos autores citados foram assinalados pelo Manual AIS-74 e, após isso, várias revisōes da AIS foram feitas, além de ter havido avarıços na assistência ao traumatizado ${ }^{1 / 4}$.

Por outro lado, é difícil comparar freqüências de mortos entre estudos mais recentes, devido a variações nos critérios de inclusão dos pacientes e também pela ausência de uniformidade nos intervalos de agrupamento dos escores ISS.

Assim, de forma geral, pode-se constatar no presente estudo que o nível de gravidade do trauma foi proporcionalmente maior nos pacientes de trauma penetrante (média do ISS $=20,8$ ) do que nos de trauma fechado (média do ISS $=14,9$ ). A mortalidade foi equivalente nos dois grupos, visto que ambos ficaram próximos a $12,0 \%$, valor este compatível

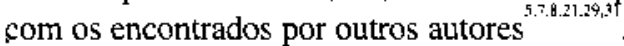

O prognóstico de pacientes de trauma é influenciado por vários fatores, tais como idade, condição

\section{Referências Bibliográficas}

1. THE ABBREVIATED INJLRY SCALE (AIS): 1990 revision. Des Plaines. Association for the Advancement of Automotive Medicine. 1990.

2. THE ABBREVIATED INJLRY SCALE (AIS): 1985 revision. Des Plaines, American Association for Automotive Medicine, 1985.

3. BAKER, S.P. et al. The Injury Sererity Score: a method for describing patients with multiple injuries and evaluating emergency care. J.Tratma, 14: 187-96, 1974.

4. BOYD. C.R. et al. Evaluating trauma care: the TRISS method. J.Trauma, 27: 370-8. 1987

5. BLLL. J.P. The Injury Severity Score of road traffic casualties in relation to mortality, time of death, hospical treatment time and disability: Accid.Anal.Pret. 7: 249-55. 1975

6. CAYTFN, C.G.\& FVA. $5, W$. Severity indices and their implications for emergency medical services research and evaluation. J.Trauma. 19: 98-102, 1979. de saúde pré-injúria, tempo de decorrido entre o evento e a assistência definitiva, qualidade da assistência e complicaçōes ${ }^{7,17,36}$.Entretanto o tipo e a gravidade das lesöes, aspectos levados em consideração pela AIS e consequientemente refletidos no ISS, têm sido reconhecidos como os fatores mais importantes na determinação prognóstica ${ }^{1 ?: 3.30}$.

Enquanto o tipo de lesão pode ser definido através de diagnósticos médicos, a gravidade não tem formulação concordante uma vez que pode ter significados distintos para diferentes pessoas. Pode-se inferir por gravidade a extensão das injúrias, o risco que elas causam à vida, o período e o grau de incapacitação bem como a proporção de incapacidades permanentes. Entretanto, quando se tratam de lesōes graves, há pouca discordância de que o critério principal de julgamento da gravidade é o risco para a vida ${ }^{30}$.

Determinar a proporção de feridos, incapacitados e mortos e, também. identificar os tipos de lesōes apresentadas pelas vítimas de trauma, de acordo com cada tipo de acidente ou violência, é fundamental para se elaborar o perfil desses eventos e melhor fundamentar as medidas preventivas de um país ou mesmo de uma comunidade. Todavia, para que haja avanço no conhecimento a respeito do trauma é imperativo o estudo da gravidade das lesões e da gravidade do trauma. Tais estudos se tornaram possíveis com o desenvolvimento dos índices de gravidade.

Espera-se que o presente estudo sirva de estímulo para a realização de outras pesquisas a respeito dos índices de gravidade.

7 CHAYPION. H.R et al. Hajor trauma in geriatric patients. Aml.Public Health. 79: $1278-82$ 1989.

8. CHAMPION, H.R et al. The major trauma outcome study: establishing national nornss for trauma care. J.Trauma. 30: 1356-65. 1990.

9. CHAMPION. H.R. et al. A neN characterization of injury severity. J.7iama 30: 539-45. 1990.

10. CHAMPION- H.R. el al. A revision of the trauma score. J.Trauma. 29: 623-9. 1989.

11. CHAMPION, H.R. et al. Trauma scoring. In: Moore. E.E. et al. Tratma. 2.ed. California. Appleton \& Lange. 1991. p.47-65.

12. CHAMPION. H.R. et al. Trauma severity scoring to predict mortality. Wonld J.Strg. 7: 4-11, 1983.

13. CIVIL, I.D. \& SCHWAB, C.W. The Abbreviated Injury Scale. 1985 revision: a condensed chart for clinical use. J.Trama. 28: 87-90. 1988.

14. COPES. W.S. et al. The Injury Severity score revisited. J.Tratma. 28: 69-?․ I988. 
15. CLRRENT status of trauma severity indices. J.Trama. 23: 185-98, 1983.

16. DALOSSI. T. Dereminaçào precoce do nivel de gravidade do trauma. Săo Paulo. 1993. [Dissertação de Mestrado - Escola de Enfermagem da [-SP].

17. FRLTIGER. A. et al. Five years follow-up of severely injured ICL patients. J.Trama, 31: 1216-25. 1991

18. GENXARELLI. T.A. et al. Hortality of patients with head injury and extracranial injury treated in trauma centers. ,Tratma. 29: 1193201. 1989.

19. GORMICAX, S.P. CRAMS scale: field triage of trauma rictims. Ann. Fmeng..lfed. 11: 132-5. 1982.

20. GREENSPAN, L. et al. Abtreviated Injury Scale and Injury Severity Score: a scoring chart. J.Tratma. 25: 60-4. 1985.

21 HILL. A.B. et al. Chest trauma in a canadian urban setting: implications for trauma research in Canada. J.Trauma. 31: 971-3, 1991.

22. KOIZLMI, M.S. Aspectos epidemiológicos dos acidentes de motociclera no Município de Sào Paulo, 1982. Sào Paulo, 1984. [Tese de Doutorado - Faculdade de Saúde Pública da LSP].

23. KOIZLMI, M.S. Natureza das lesòes nas vítimas de acidentes de motocicleta. São Paulo. 1990. [Tese de Livre-Docêncià - Escola de Enfermagem da $L S P]$.
24. KRISCHER. J.P. Indexes of severity: conceptual development. Health Serv'Res. 14: 56-6?. 1979.

25. LEVY. P.S et al. The revised estinated survival probability index of trauma severity. Public Health Rep. 97: 452-9. 1982.

26. LOXG. W.B. et aI. Accuracy and relationship of mecanisms of injury. trauma score. and injury severity score in identifying major trauma. Am.J.Sug. 151: 581-4, 1986.

27. MORG4N, T.O. et al. Injury severity scoring: influence of timing and nurse raters on accuracy. Heant Lthig. 17: 256-61. 1988.

28. RATIXG the severity of tissue damage: the Abbreviated Injury Scale - part 1. JA $L A$. 215: 27?-80. 19?1.

29. SCHWAB. C.W. et al. DRG reinbursement for trauma: the demise of the trauma center the use of ISS grouping as an early predictor of total hospital cost). J.Tratma. 28: 939-45. 1988.

30. SF.MILOW, J.L \& CONE. R. Ltility of the injury severity score: a confirnation. Health Sen'.Res. 11: $45-52.19^{-} 6$.

31. SMITH, D.P. et al. Trauma in the elderly: determinants of outcome. South.lHed.f. 83:17:1-7. 1990.

32. THOMPSON, J. \& DAMS, J. Indices of injur: development and status. Nitr. Clin. Worth Am. 21: 655-7?. 1986.

\begin{abstract}
The severity of traumas in hospitalized patients was characterized by means of the injury Severity Score (ISS) was studied prospectively. One hundred trauma patients hospitalized in a trauma referral health facility located in $S$. Paulo, Brazit, were analyzed. Of the total number of patients, 68 had blunt trauma and 32 penetrating trauma. As to ISS, if was discovered that $53.0 \%$ of the blunt trauma patients had mild trauma (ISS $1-15$ ), $29,4 \%$ moderate trauma (ISS 16 24), and $17.6 \%$ showed severe trauma (ISS 25); 34.4\% of the penetrating trauma patients had mild trauma, $18.7 \%$ moderate trauma and $46.9 \%$ severe trauma. The mean and standard deviations relationg to the ISS of blut and penetrating trauma patients were, respectively, $14.9 \pm 8.1$ and $20.8 \pm 11.0$; the respective mortality rates were of $11.8 \%$ and $12.5 \%$
\end{abstract}

Injury Severity Score. Wounds and injuries, prevention and control. 
Anexo 1

CAIS - 85 F

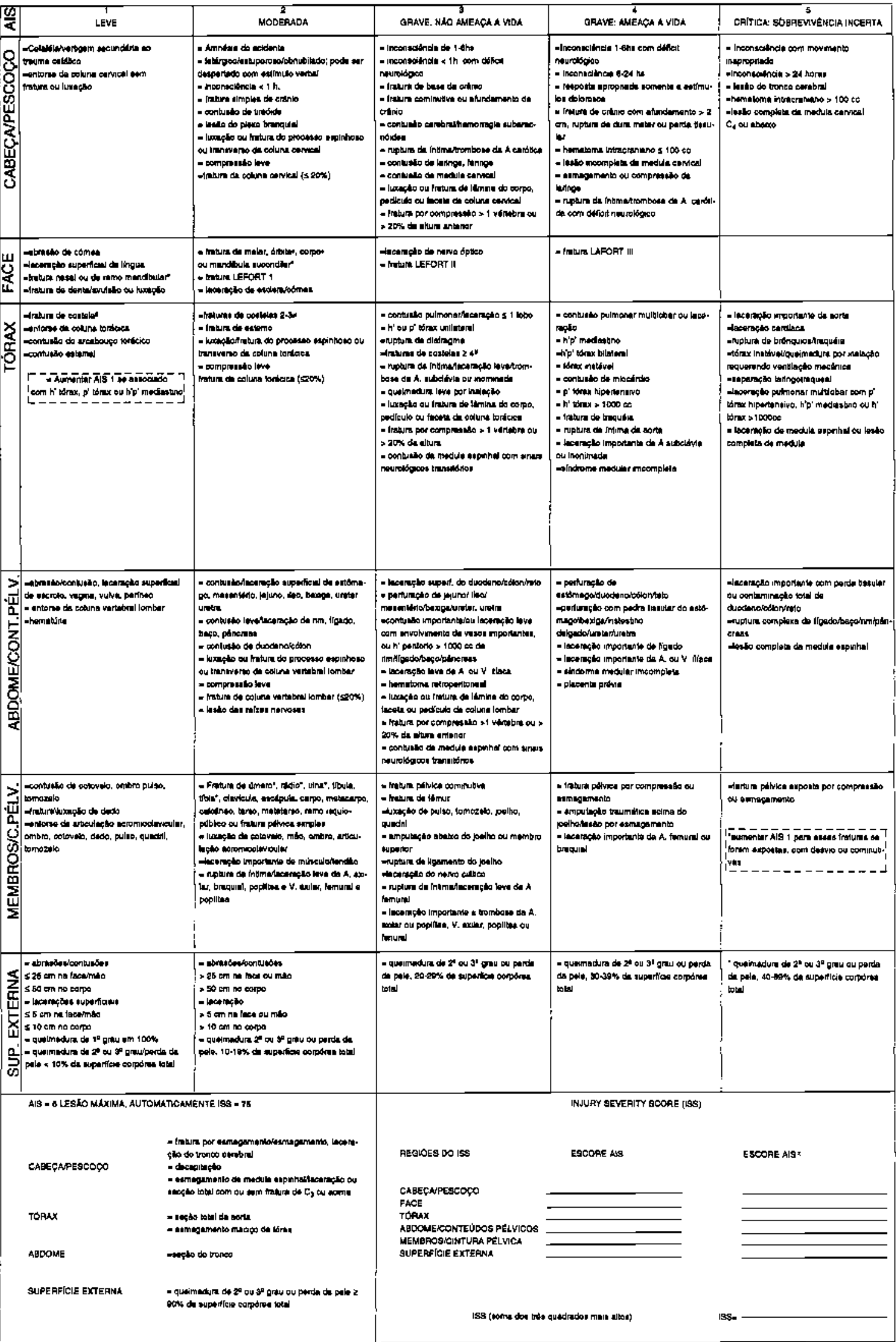


Anexo 2

CAIS - 85 P

\begin{tabular}{|c|c|c|c|c|c|}
\hline$\frac{01}{4}$ & $\begin{array}{c}1 \\
\text { LEVE }\end{array}$ & MOOERADA & $\begin{array}{c}3 \\
\text { GAAVE NAO AMEAGA A VIDA }\end{array}$ & GAAYE AMEACQA A VIDA & $\begin{array}{c}5 \\
\text { CRIJICA LOBFEVIVENCIM INCERTA }\end{array}$ \\
\hline 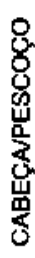 & 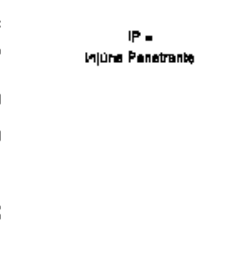 & 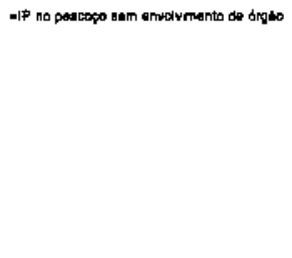 & 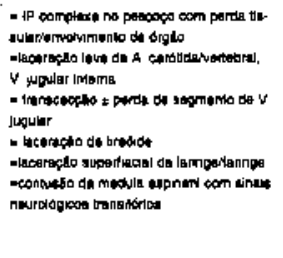 & 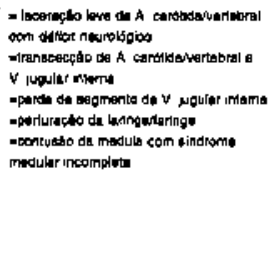 & 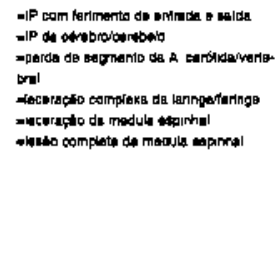 \\
\hline 岂 & IP sam pords ossoular & 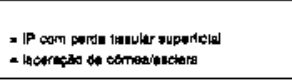 & 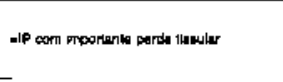 & & \\
\hline 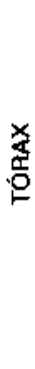 & 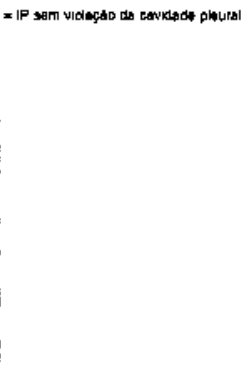 & 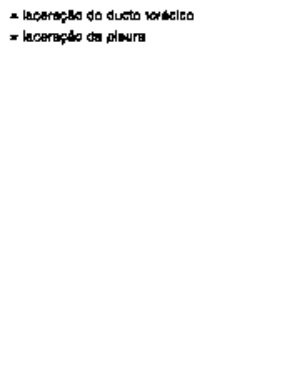 & 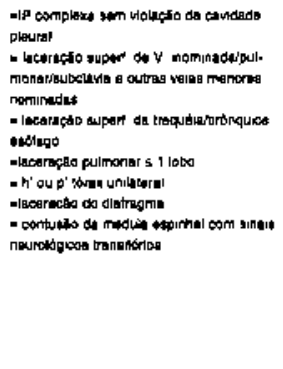 & 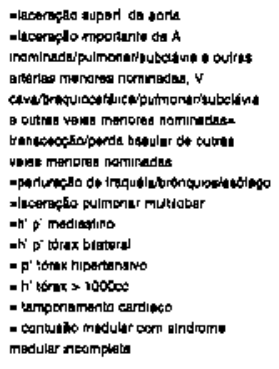 & 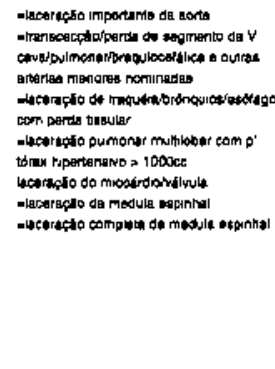 \\
\hline
\end{tabular}

("Condensed Abrevlatted Injury Scale" - Penetrante) 